\title{
Combined fit of the energy spectrum and mass composition across the ankle with the data measured at the Pierre Auger Observatory
}

\author{
Eleonora Guido ${ }^{a, *}$ on behalf of the Pierre Auger ${ }^{b}$ Collaboration \\ (a complete list of authors can be found at the end of the proceedings) \\ ${ }^{a}$ INFN and Università degli Studi di Torino, Via Pietro Giuria 1, 10125 Torino, Italy \\ ${ }^{b}$ Observatorio Pierre Auger, Av. San Martín Norte 304, 5613 Malargüe, Argentina \\ E-mail: spokespersons@auger.org
}

\begin{abstract}
The combined fit of the energy spectrum and mass composition data above $5 \cdot 10^{18} \mathrm{eV}$ suggested the presence of extragalactic sources ejecting ultra-high-energy cosmic rays with relatively low maximum energies, hard spectral indices and mixed chemical compositions, dominated by the contribution of intermediate mass groups. Here we present an extension of the fit to lower energies, to include the feature observed near $5 \cdot 10^{18} \mathrm{eV}$ in the all-particle energy spectrum, the so-called ankle. We show that it is possible to generate such a change of slope assuming that the flux below the ankle is provided by the superposition of different contributions. The simplest extension of this sort consists of introducing a supplemental extragalactic component at low energy, characterised by different physical parameters with respect to the one being dominant above the ankle: such a component may originate from a different population of sources or be provided by interactions occurring in the acceleration sites. In this framework we also explore the possibility of including the end of a Galactic contribution at low energies. The fit suggests that these scenarios provide a reasonable description of the measurements across the ankle, without significantly affecting the interpretation obtained for the above-ankle region.

In order to evaluate our capability to constrain the source models, we finally discuss the impact of the main experimental systematic uncertainties and of the theoretical models choice on the fit results.
\end{abstract}

$37^{\text {th }}$ International Cosmic Ray Conference (ICRC 2021)

July 12th - 23rd, 2021

Online - Berlin, Germany

\footnotetext{
${ }^{*}$ Presenter
} 


\section{Introduction}

The existence of ultra-high-energy cosmic rays (UHECRs), the ones reaching Earth with energies above $\sim 10^{18} \mathrm{eV}$, was proven in the early 1960s and recent measurements point to a predominant flux component of extragalactic origin at these energies [1]. In the still open quest for the sources of these particles, the large ground-based experiments built in the last few decades, like the Pierre Auger Observatory, have been helping in shedding light on such open questions.

In this analysis we simultaneously fit a simple astrophysical model to both the energy spectrum and the mass composition data measured at the Pierre Auger Observatory, considering energies from $10^{17.8} \mathrm{eV}$ to include the region across the ankle. At this first stage, the effects of the potentially relevant interactions occurring in the acceleration sites are not considered, limiting the study to constrain the physical parameters related to the energy spectrum and the mass composition of particles escaping the environments of extragalactic sources. In a previous publication [2], a model consisting of one single population of extragalactic sources was fitted to the data above the ankle $\left(E>10^{18.7} \mathrm{eV}\right)$. Here, since we want to interpret also the ankle region, we assume the presence of one (or more) additional contribution(s) at low energies, so that the ankle feature results from the superposition of different components. Each extragalactic component originates from a population of identical sources, uniformly distributed in the comoving volume except for a local overdensity for distances smaller than $\sim 30 \mathrm{Mpc}$. The overdensity is considered as a cluster centred around our Galaxy, following [3], which provides a good approximation to nearby densities if compared to the distributions of stellar mass and star formation (SF) rate over the full sky illustrated in [4]. Each component is given by the superposition of the contributions of $n \leq 5$ representative nuclear species $A$, chosen among ${ }^{1} \mathrm{H},{ }^{4} \mathrm{He},{ }^{14} \mathrm{~N},{ }^{28} \mathrm{Si},{ }^{56} \mathrm{Fe}$, ejected according to a power-law spectrum with a rigidity-dependent broken exponential cutoff:

$$
J(E)=\sum_{A} f_{A} \cdot J_{0} \cdot\left(\frac{E}{E_{0}}\right)^{-\gamma} \cdot \begin{cases}1, & E<Z_{A} \cdot R_{\text {cut }} \\ \exp \left(1-\frac{E}{Z_{A} \cdot R_{\text {cut }}}\right), & E>Z_{A} \cdot R_{\text {cut }} .\end{cases}
$$

where $J_{0}$ is the normalisation factor, $Z_{A}$ is the atomic number of each species $A$ and $f_{A}$ is the fraction of $A$ at the energy $E_{0}=10^{17.5} \mathrm{eV}$.

The energy spectrum and mass composition of the particles escaping from the sources are modified during the propagation in the intergalactic medium (IGM) by the adiabatic energy losses and the interactions with background photons. We take

\begin{tabular}{c|c|c}
$\sigma_{\mathrm{pd}}$ & Talys [6], PSB [7] & $\mathbf{X Y Z}$ \\
EBL & Gilmore [8], Dominguez [9] & XYZ \\
HIM & EPOS-LHC [10], Sibyll2.3d [11], QGSJetIIv4 [12] & XYZ
\end{tabular}

Table 1: The propagation models used in this analysis. The bold letters define the label 'XYZ'. For instance, 'TGE' stands for Talys, Glimore and EPOS-LHC models. into account these effects by using SimProp [5] simulations, where the uncertain quantities, i.e. the photodisintegration cross sections $\sigma_{\mathrm{pd}}$ and the EBL spectrum, are treated with phenomenological models. Besides, since a direct measurement of the mass composition is not possible on an event-by-event basis, we use the distribution of $X_{\max }$ as an estimator of the mass distribution in each energy bin. The conversion to the mass distribution depends on the chosen hadronic interaction model (HIM), which is thus another source of uncertainty. The various propagation models used in this analysis are shown in Tab. 1. We choose the configuration labelled as "TGE" as our reference and the impact of the models on the fit results will be discussed in Sec. 4 . 


\section{Fit procedure and results}

We use a measurement of the energy spectrum in $\log _{10}(E / \mathrm{eV})$ bins of 0.1 width from $10^{17.8} \mathrm{eV}$ to $10^{20.2} \mathrm{eV}$, obtained with the data collected over 15 years with the Surface Detector Array of the Observatory [14]. As for the $X_{\max }$ distributions, measured by the fluorescence telescopes, we consider $\log _{10}(E / \mathrm{eV})$ bins of 0.1 from $10^{17.8} \mathrm{eV}$ to $10^{19.6} \mathrm{eV}$ and one additional larger bin containing events with energies greater than $10^{19.6} \mathrm{eV}$; each $X_{\max }$ distribution is binned in intervals of $20 \mathrm{~g} \mathrm{~cm}^{-2}$ [15]. In the fit we minimise the deviance $D=-\ln \left(L / L_{\mathrm{sat}}\right)$, a generalised $\chi^{2}$, where $L$ is our model and $L_{\text {sat }}$ is a model that perfectly describes the data. It consists of two terms, $D_{J}$ and $D_{\mathrm{X}_{\max }}$. The first is for the energy spectrum and is a product of Gaussian distributions. The latter is a product of multinomial distributions used for the fit of the $X_{\max }$ distributions; they are modelled as Gumbel distribution functions [16], whose parameters depend on the hadronic interaction model.

For each extragalactic component the free fit parameters are the luminosity density $\mathcal{L}_{0}(E>$ $10^{17} \mathrm{eV}$ ), the spectral index $\gamma$, the rigidity cutoff $R_{\text {cut }}$ and $n-1$ of the $n$ mass fractions $f_{A}{ }^{1}$. Since the mass fractions are extrapolations of the mass composition at a fixed energy lower than the fit threshold, they are not necessarily very informative about the actual composition in the energy range involved in our fit, especially if the energy spectrum is very hard; thus we express the mass composition in terms of the fractions of the energy density integral $I_{A}^{2}$ above $E_{\min }=10^{17}$.

\begin{tabular}{|c|c|c|c|c|}
\hline Galactic contribution (at Earth) & \multicolumn{2}{|c|}{$\mathrm{N}+\mathrm{Si}$} & \multicolumn{2}{|c|}{-} \\
\hline$J_{0}^{\mathrm{gal}}\left[\mathrm{eV}^{-1} \mathrm{~km}^{-2} \mathrm{sr}^{-1} \mathrm{yr}^{-1}\right]$ & \multirow{2}{*}{\multicolumn{2}{|c|}{$\begin{array}{c}(1.07 \pm 0.06) \cdot 10^{-13} \\
17.48 \pm 0.02 \\
93.0\end{array}$}} & \multirow{2}{*}{\multicolumn{2}{|c|}{$\begin{array}{l}- \\
- \\
-\end{array}$}} \\
\hline $\begin{array}{l}\log _{10}\left(R_{\mathrm{cut}}^{\mathrm{gal}} / \mathrm{V}\right) \\
f_{\mathrm{N}}(\%)\end{array}$ & & & & \\
\hline EG components (at the sources) & Low energy & High energy & Low energy & High energy \\
\hline $\mathcal{L}_{0}\left[\mathrm{erg} \mathrm{Mpc}^{-3} \mathrm{yr}^{-1}\right]$ & $7.28 \cdot 10^{45}$ & $4.4 \cdot 10^{44}$ & $1.7 \cdot 10^{46}$ & $4.5 \cdot 10^{44}$ \\
\hline$\gamma$ & $3.30 \pm 0.05$ & $-1.47 \pm 0.12$ & $3.49 \pm 0.02$ & $-1.98 \pm 0.10$ \\
\hline $\log _{10}\left(R_{\text {cut }} / \mathrm{V}\right)$ & 24 (lim.) & $18.19 \pm 0.02$ & 24 (lim.) & $18.16 \pm 0.01$ \\
\hline$I_{\mathrm{H}}(\%)$ & 100 (fixed) & 0.0 & 49.87 & 0.0 \\
\hline$I_{\mathrm{He}}(\%)$ & - & 27.17 & 10.92 & 28.60 \\
\hline$I_{\mathrm{N}}(\%)$ & - & 69.86 & 36.25 & 69.05 \\
\hline$I_{\mathrm{Si}}(\%)$ & - & 0.0 & 0.0 & 0.0 \\
\hline$I_{\mathrm{Fe}}(\%)$ & - & 2.97 & 2.96 & 2.35 \\
\hline$D_{J}\left(N_{J}\right)$ & & (24) & & (24) \\
\hline$D_{X_{\max }}\left(N_{X_{\max }}\right)$ & 593 & (329) & & (329) \\
\hline$D(N)$ & & & & (353) \\
\hline
\end{tabular}

Table 2: The fit results in the TGE configuration. Below the ankle we considered an extragalactic component of pure protons together with a heavier Galactic contribution (left) or a mixed extragalactic component (right).

The above-ankle data are reproduced by a mixed component where all the $n=5$ representative masses are allowed at the sources. As for the low-energy region, i.e. the one below the ankle, we consider two scenarios and the best fit results are summarised in Tab. 2. In the first scenario we use a second extragalactic component of pure protons and an additional Galactic contribution at Earth, given by a power law with $\gamma=3.2$ modified by an exponential cutoff. Several mass compositions have been considered for such a Galactic contribution, both pure and mixed, and the lowest deviance is obtained by assuming a composition dominated by medium-mass nuclei (e.g. nitrogen), with a small contribution of heavier species (e.g. silicon); the fraction of nitrogen $\left(f_{N}\right)$ at the normalisation energy $E_{0}^{\mathrm{gal}}=10^{16.85} \mathrm{eV}$, the normalisation $J_{0}^{\mathrm{gal}}$ and the $Z$-dependent rigidity cutoff $\log _{10}\left(R_{\mathrm{cut}}^{\mathrm{gal}}\right)$

${ }^{1}$ The $n$-th mass fraction is obtained from the requirement that the sum $\sum_{A} f_{A}$ is equal to one.

${ }^{2}$ The integral of the energy density above a given energy $E_{\min }$ for a nuclear species $A$ is defined as $\int_{E_{\min }}^{\infty} f_{A} J(E) E d E$. 
are free fit parameters. Our data cannot be described by a Galactic contribution with heavier mass compositions, e.g. the deviance reaches $\sim 1000$ if a composition dominated by silicon is assumed. In the second scenario we assume only one additional mixed extragalactic component at low energies, similar to the above-ankle one, but characterised by different physical parameters. Even if this scenario exhibits a lower deviance, the difference is comparable to the systematic uncertainties effect illustrated in the next sections; in the future a more detailed investigation of the assumptions on the Galactic contribution could possibly help to establish a favoured scenario.

In both the scenarios the high-energy (HE) component exhibits a very hard energy spectrum at the sources, a relatively low maximum rigidity and a mixed mass composition, dominated by medium-mass nuclei. On the other hand, the additional low-energy (LE) extragalactic component, either light or mixed, has a very soft energy spectrum and a very high rigidity cutoff, which are also related to a larger estimated source emissivity with respect to the one of the HE component; the fit is actually degenerate with respect to $R_{\text {cut }}$ for values above $\sim 10^{19} \mathrm{eV}$, thus fixing this parameter to an arbitrarily high value, such as $10^{24} \mathrm{eV}$, provides the same best fit results.
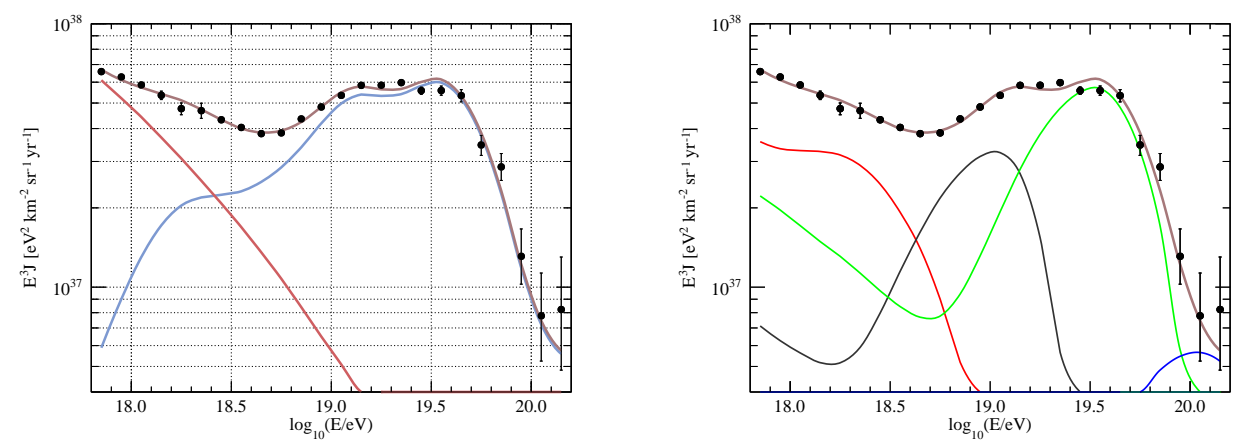

Figure 1: The measured energy spectrum and the estimated best fit results in the scenario with two mixed extragalactic components. Left: the estimated contributions from the two extragalactic components (red: LE component, blue: HE component). Right: the partial fluxes related to different nuclear species at the top of atmosphere, grouped according to their mass number: $A=1$ (red), $2 \leq A \leq 4$ (grey), $5 \leq A \leq 22$ (green), $23 \leq A \leq 38$ (cyan), $A \geq 39$ (blue).
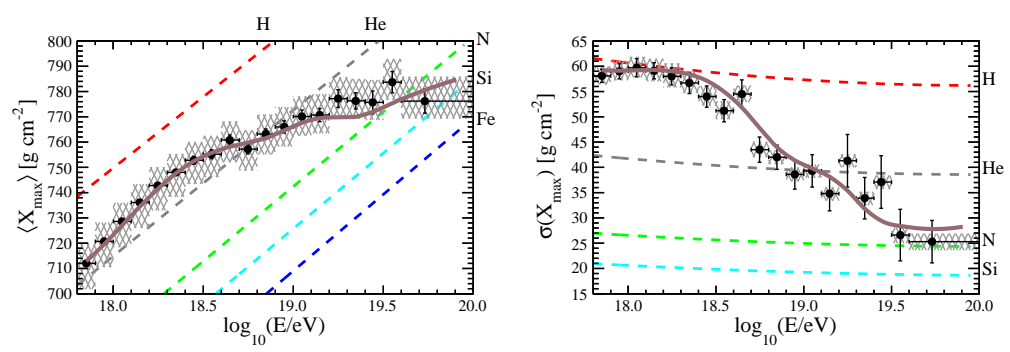

Figure 2: The first two moments of the $X_{\max }$ distributions in each energy bin along with their expected values and the predictions for pure compositions of ${ }^{1} \mathrm{H}$ (red), ${ }^{4} \mathrm{He}$ (grey), ${ }^{14} \mathrm{~N}$ (green), ${ }^{28} \mathrm{Si}$ (cyan), ${ }^{56} \mathrm{Fe}$ (blue).

In Fig. 1 and in Fig. 2 the best fit results obtained in the scenario with two mixed extragalactic components are shown with the observed energy spectrum and the first two moments of the measured $X_{\max }$ distributions. The observed mass composition below the ankle is mixed and dominated by protons and medium-mass nuclei, such as nitrogen. Above the ankle the contributions from the 
different mass groups have small overlap and the composition becomes heavier as the energy increases. The estimated non-negligible $\mathrm{Fe}$ fraction at the sources is actually required only by the energy spectrum fit, since it contributes at the highest energies where the mass composition data are not available, as already noted in [17].

\section{Effect of the experimental systematic uncertainties}

The systematic uncertainties of instrumental origin affect both the energy and the $X_{\max }$ measurements. The uncertainty on the energy scale is assumed to be $\Delta E / E=14 \%$ in the whole considered energy range [18]. For the $X_{\max }$ scale we consider an asymmetric and slightly energydependent uncertainty, ranging from 6 to $9 \mathrm{~g} \mathrm{~cm}^{-2}$ [13]. An additional systematic effect could also arise from the uncertainties on the $X_{\max }$ resolution and acceptance parameters [13], but we verified that their impact on the fit results is here negligible.

Following the same approach used in [2], we take into account the uncertainty on the energy scale and on the $X_{\max }$ scale by shifting all the measured energies and $X_{\max }$ values by one systematic standard deviation in each direction. We consider all the possible combinations of these shifts and their effect on the deviance value is summarised in Tab. 3. The dominant effect in terms of predictions at Earth is the one arising from the $X_{\max }$ uncertainty; as for the estimated best fit parameters, they are not much modified when the experimental systematic uncertainties

\begin{tabular}{cc|ccc}
$\Delta X_{\max }$ & $\Delta E / E$ & $D_{J}$ & $D_{X_{\max }}$ & $D$ \\
\hline \multirow{3}{*}{$-1 \sigma_{\text {syst }}$} & $-14 \%$ & 52.5 & 578.3 & 630.9 \\
& 0 & 71.7 & 595.2 & 666.9 \\
& $+14 \%$ & 64.9 & 609.3 & 674.2 \\
\hline \multirow{2}{*}{0} & $-14 \%$ & 53.5 & 581.3 & 634.8 \\
& 0 & 60.1 & 554.8 & 614.9 \\
& $+14 \%$ & 70.6 & 548.8 & 619.5 \\
\hline \multirow{2}{*}{$+1 \sigma_{\text {syst }}$} & $-14 \%$ & 79.1 & 714.2 & 793.3 \\
& 0 & 80.8 & 555.4 & 736.2 \\
& $+14 \%$ & 82.4 & 615.7 & 698.2 \\
\hline \multicolumn{2}{c}{}
\end{tabular}

Table 3: The effect on the deviance of the $\pm 1 \sigma_{\text {syst }}$ shifts in the energy and $X_{\max }$ scales. are considered.

The maximal variations on the predicted fluxes at Earth, obtained by considering all the configurations of Tab. 3, are shown in Fig. 3. The rather large uncertainty on the predicted total fluxes (brown band) is due to the $\pm 14 \%$ shifts in the energy scale, but it significantly affects only
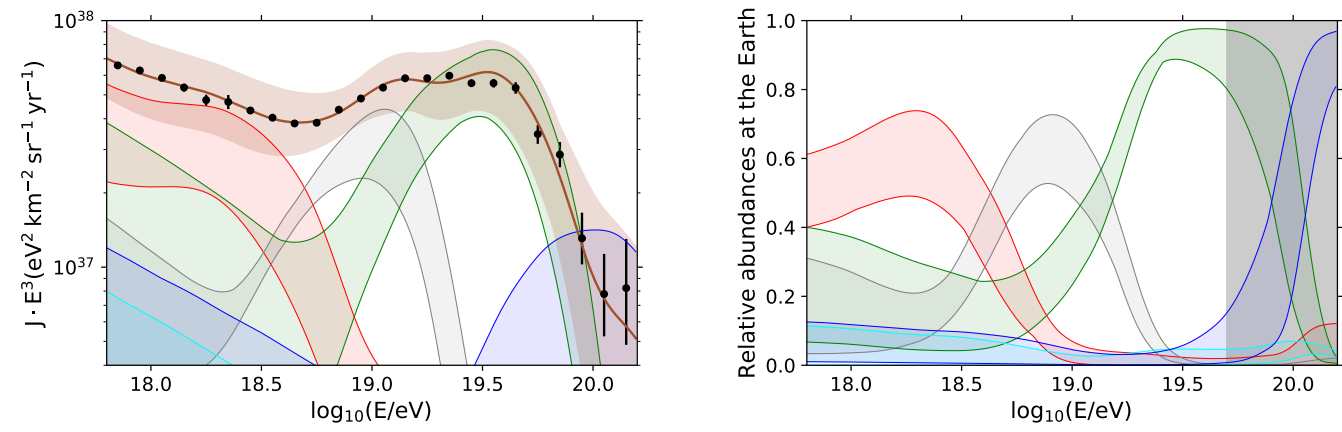

Figure 3: Left: the combined effect of the experimental uncertainties on the energy spectrum. Right: the effect on the relative abundances at the top of atmosphere. The uncertainties are considered by shifting the energies and/or the $X_{\max }$ distributions of $1 \sigma_{\text {syst }}$ in both directions, as shown in Tab. 3. The bands represent the maximal variations induced by considering all the possible combinations of shifts. The shaded area in the right plot indicates the region where the $X_{\max }$ measurements are grouped in one single energy bin because of the low statistics and thus the mass composition predictions are mainly driven by the energy spectrum fit. 
the estimated source emissivities (the total variation of $\mathcal{L}_{0}$ is of $\sim 11 \cdot 10^{45} \mathrm{erg} \mathrm{Mpc}^{-3} \mathrm{yr}^{-1}$ and $\sim 2 \cdot 10^{44} \mathrm{erg} \mathrm{Mpc}^{-3} \mathrm{yr}^{-1}$ for the LE and the HE component, respectively), whereas the description of the energy spectrum and the mass composition data is almost unchanged; the largest modifications of the predicted abundances at Earth are induced by the shifts in the $X_{\max }$ scale, which also strongly affect the deviance value.

\section{Effect of the uncertainties from models}

We also investigate the impact on the fit results of changing the propagation models and the hadronic interaction model. In all the cases we repeat the fit considering different combinations of propagation models, labelled as ' $\mathrm{XY}$ ' according to Tab. 1. The results thus obtained are written in Tab. 4 and their effect on the predicted fluxes at Earth is shown in Fig. 4.

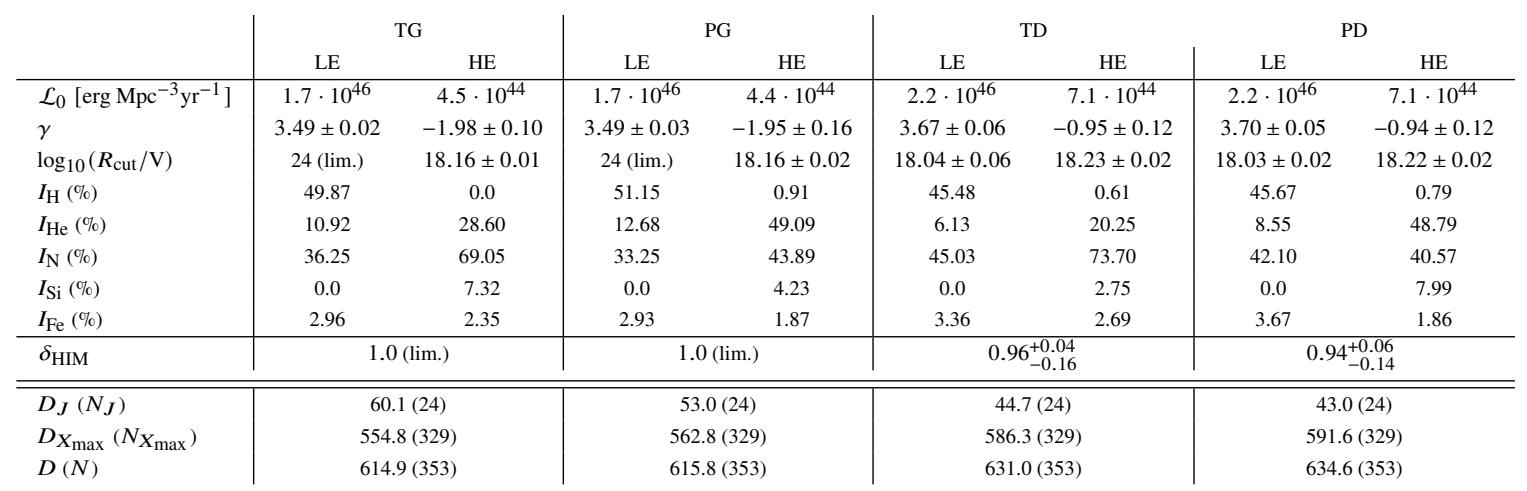

Table 4: Best fit results obtained by using different combinations of propagation models. The uncertainty due to the hadronic interaction model choice is considered by fitting the nuisance parameter $\delta_{\mathrm{HIM}}$.

As concerns the hadronic interaction model, we verified that QGSJetIIv4 cannot properly describe our data $(D>1000)$ and is thus excluded from this analysis. Since we want to keep open the option that our data are better described by an intermediate model between EPOS-LHC and Sibyll2.3d instead of exactly one of them, we introduce an additional nuisance parameter $\delta_{\mathrm{HIM}}$, limited between 0 and 1, which defines the value of each HIM-dependent Gumbel parameter as $p=\delta_{\mathrm{HIM}} \cdot p^{\mathrm{EPOS}}+\left(1-\delta_{\mathrm{HIM}}\right) \cdot p^{\mathrm{Sibyll}}$.

Our data appear to be better described by either EPOS-LHC or intermediate models compatible with it, regardless of the propagation models configuration, making the hadronic interaction model choice the dominant uncertainty among the ones from models in terms of predictions at Earth. As concerns the models for the propagation in the IGM, some expected modifications in the best fit parameters at the sources are observed if we change our assumptions on the propagation details. When the photodisintegration cross sections are modelled on PSB a larger amount of helium is ejected at the sources to compensate the absence of $\alpha$ emissions during propagation; when the EBL spectrum is based on Dominguez the LE component is suppressed at lower energy with a constrained $R_{\text {cut }}$ to compensate the larger amount of secondary particles below the ankle provided by the HE component. The lowest deviance is obtained in the TGE configuration, however the impact of changing the propagation models on the deviance and on the predicted fluxes at Earth is encompassed within the experimental systematic uncertainties. 

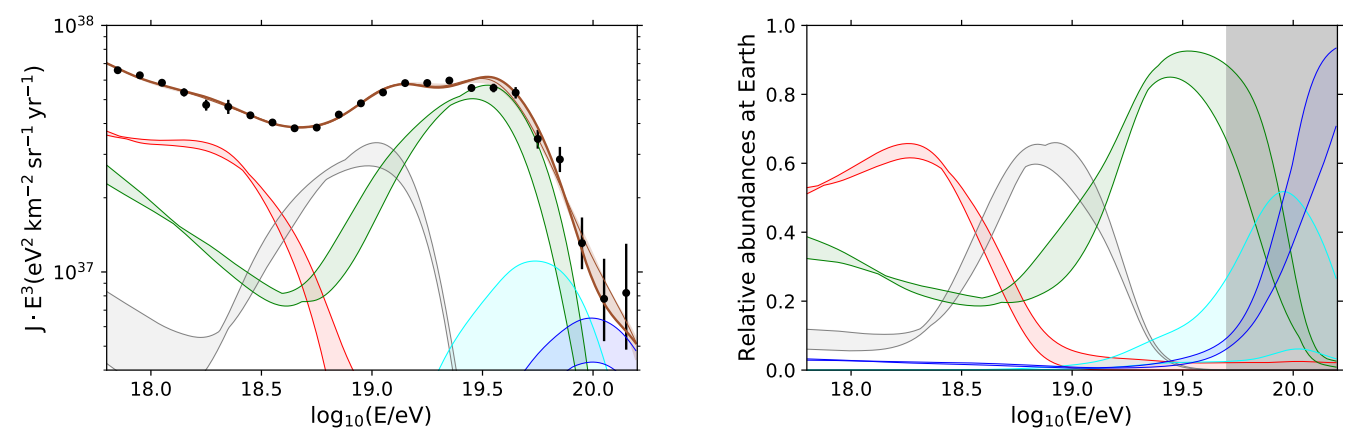

Figure 4: Left: the effect of the uncertainties from models on the energy spectrum. Right: the effect on the relative abundances at the top of atmosphere. The bands represent the maximal variations given by the results in Tab. 4 .

\section{Source evolution}

All the results presented in the previous sections are obtained by assuming no cosmological evolution for the populations of extragalactic sources. We perform the fit also assuming three different evolution scenarios: we consider a SF-like [19] evolution, an AGN-like one [20], which have a positive source evolution for $z<1$ ( $m=3.5$ and $m=5$, respectively), and a TDE-like evolution with $m=-3$ for small $z$ [21]. Since there is no physical reasons to assume that the two populations of sources have the same cosmological evolution, all the possible combinations are considered and the results in terms of total deviance are summarised in Fig. 5. In the case of the LE component, a positive (negative) evolution produces a hardening (softening) of the energy spectrum at the sources to compensate the larger amount of low (high) energy particles. As for the HE component, the cosmological evolution effect is balanced by the interplay between the modification of the energy spectrum at the sources and/or the adjustment of the rigidity cutoff of the LE component. If the HE population has a strong positive evolution (e.g. $m=5$ ), the hardening of the energy spectrum at the sources is not enough to compensate the increased amount of low-energy particles, hence the LE component is suppressed below $\sim 10^{18} \mathrm{eV}$ to

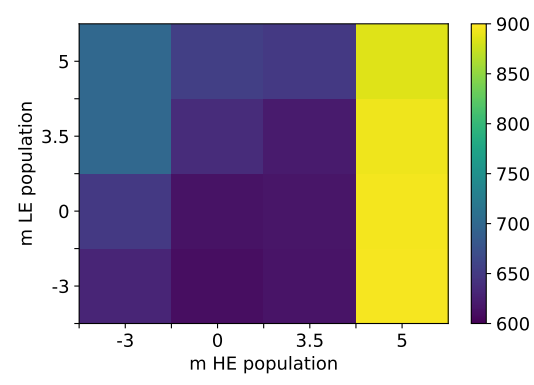

Figure 5: Deviance as a function of the cosmological evolution of the two populations.

attempt the description of the whole energy range with the HE component alone; the deviances are very high, so that such scenarios are excluded by our data at high significance. In all the other scenarios, the impact on the fit results is within the systematic uncertainties effect, so it is more difficult to draw a conclusion about a favoured configuration. However, when we consider the values $m=0,3.5$ for the HE component and $m=-3,0$ for the LE one, we obtain the lowest deviances.

\section{Conclusions}

In this study we performed a combined fit of the energy spectrum and mass composition data above $\sim 6 \cdot 10^{17} \mathrm{eV}$. The region above the ankle is described by an extragalactic component escaping from the sources with a very hard energy spectrum $(\gamma<0)$, a rather low rigidity cutoff and a mass 
composition dominated by medium-mass elements. For values of the rigidity cutoff lower than approximately $10^{18.5} \mathrm{~V}$, such as in the fits shown here, source effects contribute to the observed suppression of the spectrum at the highest energies more than propagation energy losses, though neither is negligible. The negative spectral index produces very hard elemental fluxes at Earth, not much superposed, which describe the very pronounced spectral features of the measured energy spectrum and the rather narrow $X_{\max }$ distributions. A best fit solution with a spectral index $\leq 1$ was already favoured in the case of the above-ankle fit presented in [2]. The subsequent energy spectrum hardening is actually comparable to the effects of the systematic uncertainties, since the deviance profile is approximately flat for $R_{\text {cut }} \leq 5 \cdot 10^{18} \mathrm{eV}$ and $\gamma \leq 1$.

As concerns the data below the ankle, the presence of a Galactic heavy mass contribution is disfavoured. Instead, our data can be described by an additional light or light-to-intermediate very soft extragalactic component, with the presence of a medium-mass Galactic component in the first case or without it in the latter. Taking into account the photodisintegration processes in the acceleration sites [22] or considering non-identical sources with different maximal energies [23] could explain an observed very soft component of protons or mixed elements, respectively.

Among the experimental systematic uncertainties, the strongest effect on the deviance value and on the predicted fluxes at Earth comes from the uncertainty on the $X_{\max }$ scale. As for the uncertainties from models, their impact is smaller; however, our data are generally better described when EPOS-LHC is used rather than the other post-LHC models. It is worth noting that the Observatory, thanks to its low-energy enhancements, can also detect events below $6 \cdot 10^{17} \mathrm{eV}$, thus in the next future the lower-energy data sets could be included as well, with the aim also to improve the understanding of the Galactic to extragalactic transition region.

\section{References}

[1] A. Aab et al. [Pierre Auger Coll.], Astrophys. J., 891 (2020) 142.

[2] A. Aab et al. [Pierre Auger Coll.], J. Cosmol. Astropart. P., 04 (2017) 038.

[3] J. J. Condon et al. Astrophys. J. 872 (2019) 148.

[4] J. Biteau (2021) [2105.11345].

[5] R. Aloisio et al., J. Cosmol. Astropart. P., 11 (2017) 009.

[6] A.J. Koning et al., International Conference on Nuclear Data for Science and Technology, 058 (2008).

[7] J. Puget et al., Astrophys. J., 205 (1976) 638-654.

[8] R. Gilmore et al., Mon. Not. R. Astron. Soc., 422 (2012) 3189-3207.

[9] A. Dominguez et al., Mon. Not. R. Astron. Soc.,410 (2011) 2556-2578.

[10] T. Pierog at al., Phys. Rev. C, 92 (2015) 034906.

[11] F. Riehn et al., Phys. Rev. D, 102 (2020) 6, 063002.

[12] S. Ostapchenko, Phys. Rev. D, 83 (2011), 014018.

[13] A. Aab et al., Phys. Rev. D, 90 (2014) 12, 122005.

[14] A. Aab et al., to be submitted to Eur. Physical J. Conf.

[15] A. Yushkov [for the Pierre Auger Coll.], Proc. 36th Int. Cosmic Ray Conf. (2019), PoS(ICRC2019)482.

[16] M. De Domenico et al., J. Cosmol. Astropart. P., 07 (2013) 07, 050.

[17] J. Heinze et al., Astrophys. J., 873 (2019) 1, 88.

[18] A. Aab et al. [Pierre Auger Coll.], Phys. Rev. D, 102 (2020) 062005.

[19] H. Yuksel et al., Astrophys. J., 683 (2008) L5-L8 .

[20] G. Hasinger et al., Astron. Astrophys., 441 (2005) 417-434.

[21] D. Biehl et al., Sci. Rep., 8, (2018) 10828.

[22] M. Unger et al., Phys. Rev. D, 92 (2015), 123001.

[23] M. Kachelriess et al., Phys. Lett. B, 634 (2006) 143-147. 


\section{The Pierre Auger Collaboration}

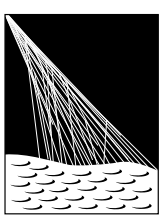

PIERRE

AUSGERVATORY

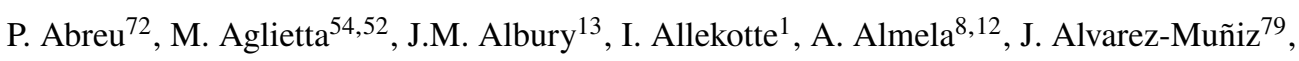
R. Alves Batista ${ }^{80}$, G.A. Anastasi ${ }^{63,52}$, L. Anchordoqui ${ }^{87}$, B. Andrada ${ }^{8}$, S. Andringa ${ }^{72}$, C. $\mathrm{Aramo}^{50}$, P.R. Araújo Ferreira ${ }^{42}$, J. C. Arteaga Velázquez ${ }^{67}$, H. Asorey $^{8}$, P. Assis ${ }^{72}$, G. Avila ${ }^{11}$, A.M. Badescu ${ }^{75}$, A. Bakalova ${ }^{32}$, A. Balaceanu ${ }^{73}$, F. Barbato ${ }^{45,46}$, R.J. Barreira Luz $^{72}$, K.H. Becker ${ }^{38}$, J.A. Bellido ${ }^{13,69}$, C. Berat ${ }^{36}$, M.E. Bertaina ${ }^{63,52}$, X. Bertou ${ }^{1}$, P.L. Biermann ${ }^{b}$, V. Binet ${ }^{6}$, K. Bismark ${ }^{39,8}$, T. Bister ${ }^{42}$, J. Biteau ${ }^{37}$, J. Blazek ${ }^{32}$, C. Bleve ${ }^{36}$, M. Boháčová ${ }^{32}$, D. Boncioli ${ }^{57,46}$, C. Bonifazi ${ }^{9,26}$, L. Bonneau Arbeletche ${ }^{21}$, N. Borodai ${ }^{70}$, A.M. Botti ${ }^{8}$, J. Brack ${ }^{d}$, T. Bretz ${ }^{42}$, P.G. Brichetto Orchera ${ }^{8}$, F.L. Briechle ${ }^{42}$, P. Buchholz ${ }^{44}$, A. Bueno ${ }^{78}$, S. Buitink ${ }^{15}$, M. Buscemi ${ }^{47}$, M. Büsken ${ }^{39,8}$, K.S. Caballero-Mora ${ }^{66}$, L. Caccianiga ${ }^{59,49}$, F. Canfora ${ }^{80,81}$, I. Caracas ${ }^{38}$, J.M. Carceller ${ }^{78}$, R. Caruso ${ }^{58,47}$, A. Castellina ${ }^{54,52}$, F. Catalanii ${ }^{19}$, G. Cataldi ${ }^{48}$, L. Cazon ${ }^{72}$, M. Cerda ${ }^{10}$, J.A. Chinellato ${ }^{22}$, J. Chudoba ${ }^{32}$, L. Chytka ${ }^{33}$, R.W. Clay ${ }^{13}$, A.C. Cobos Ceruttii ${ }^{7}$, R. Colalillo ${ }^{60,50}$, A. Coleman ${ }^{93}$, M.R. Coluccia ${ }^{48}$, R. Conceição ${ }^{72}$, A. Condorelli ${ }^{45,46}$, G. Consolati ${ }^{49,55}$, F. Contreras ${ }^{11}$, F. Convenga ${ }^{56,48}$, D. Correia dos Santos $^{28}$, C.E. Covault ${ }^{85}$, S. Dasso ${ }^{5,3}$, K. Daumiller ${ }^{41}$, B.R. Dawson ${ }^{13}$, J.A. Day ${ }^{13}$, R.M. de Almeida $^{28}$, J. de Jesús ${ }^{8,41}$, S.J. de Jong ${ }^{80,81}$, G. De Mauro ${ }^{80,81}$, J.R.T. de Mello Neto ${ }^{26,27}$, I. De Mitri ${ }^{45,46}$, J. de Oliveira ${ }^{18}$, D. de Oliveira Franco ${ }^{22}$, F. de Palma ${ }^{56,48}$, V. de Souza $^{20}$, E. De Vito ${ }^{56,48}$, M. del Río ${ }^{11}$, O. Deligny ${ }^{34}$, L. Deval ${ }^{41,8}$, A. di Matteo $^{52}$, C. Dobrigkeit ${ }^{22}$, J.C. D’Olivo ${ }^{68}$, L.M. Domingues Mendes ${ }^{72}$, R.C. dos Anjos ${ }^{25}$, D. dos Santos $^{28}$, M.T. Dova ${ }^{4}$, J. Ebr ${ }^{32}$, R. Engel ${ }^{39,41}$, I. Epicoco ${ }^{56,48}$, M. Erdmann ${ }^{42}$, C.O. Escobar ${ }^{a}$, A. Etchegoyen ${ }^{8,12}$, H. Falcke ${ }^{80,82,81}$, J. Farmer ${ }^{92}$, G. Farrar ${ }^{90}$, A.C. Fauth ${ }^{22}$, N. Fazzini ${ }^{a}$, F. Feldbusch ${ }^{40}$, F. Fenu ${ }^{54,52}$,

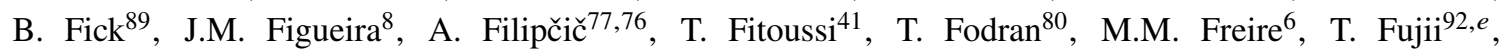
A. Fuster $^{8,12}$, C. Galea ${ }^{80}$, C. Galelli ${ }^{59,49}$, B. García ${ }^{7}$, A.L. Garcia Vegas ${ }^{42}$, H. Gemmeke ${ }^{40}$, F. Gesualdi ${ }^{8,41}$, A. Gherghel-Lascu ${ }^{73}$, P.L. Ghia ${ }^{34}$, U. Giaccari ${ }^{80}$, M. Giammarchi ${ }^{49}$, J. Glombitza ${ }^{42}$, F. Gobbi ${ }^{10}$, F. Gollan ${ }^{8}$, G. Golup ${ }^{1}$, M. Gómez Berisso ${ }^{1}$, P.F. Gómez Vitale ${ }^{11}$, J.P. Gongora ${ }^{11}$, J.M. González ${ }^{1}$, N. González ${ }^{14}$, I. Goos ${ }^{1,41}$, D. Góra ${ }^{70}$, A. Gorgi ${ }^{54,52}$, M. Gottowik ${ }^{38}$, T.D. Grubb ${ }^{13}$, F. Guarino ${ }^{60,50}$, G.P. Guedes ${ }^{23}$, E. Guido ${ }^{52,63}$, S. $\mathrm{Hahn}^{41,8}$, P. $\mathrm{Hamal}^{32}$, M.R. Hampel ${ }^{8}$, P. Hansen ${ }^{4}$, D. Harari ${ }^{1}$, V.M. Harvey ${ }^{13}$, A. Haungs ${ }^{41}$, T. Hebbeker ${ }^{42}$, D. Heck ${ }^{41}$, G.C. Hill ${ }^{13}$, C. Hojvat ${ }^{a}$, J.R. Hörandel ${ }^{80,81}$, P. Horvath ${ }^{33}$, M. Hrabovský ${ }^{33}$, T. Huege ${ }^{41,15}$, A. Insolia ${ }^{58,47}$, P.G. Isar $^{74}$, P. Janecek ${ }^{32}$, J.A. Johnsen ${ }^{86}$, J. Jurysek ${ }^{32}$, A. Kääpä ${ }^{38}$, K.H. Kampert ${ }^{38}$, N. Karastathis ${ }^{41}$, B. Keilhauer ${ }^{41}$, J. Kemp ${ }^{42}$, A. Khakurdikar ${ }^{80}$, V.V. Kizakke Covilakam $^{8,41}$, H.O. Klages ${ }^{41}$, M. Kleifges ${ }^{40}$, J. Kleinfeller ${ }^{10}$, M. Köpke ${ }^{39}$, N. Kunka ${ }^{40}$, B.L. Lago ${ }^{17}$, R.G. Lang ${ }^{20}$, N. Langner ${ }^{42}$, M.A. Leigui de Oliveira ${ }^{24}$, V. Lenok $^{41}$, A. Letessier-Selvon ${ }^{35}$, I. LhenryYvon $^{34}$, D. Lo Presti ${ }^{58,47}$, L. Lopes ${ }^{72}$, R. López ${ }^{64}$, L. Lu ${ }^{94}$, Q. Luce ${ }^{39}$, J.P. Lundquist ${ }^{76}$, A. Machado Payeras $^{22}$, G. Mancarella ${ }^{56,48}$, D. Mandat ${ }^{32}$, B.C. Manning ${ }^{13}$, J. Manshanden ${ }^{43}$, P. Mantsch ${ }^{a}$, S. Marafico ${ }^{34}$, A.G. Mariazzi ${ }^{4}$, I.C. Mariş ${ }^{14}$, G. Marsella ${ }^{61,47}$, D. Martello ${ }^{56,48}$, S. Martinelli ${ }^{41,8}$, O. Martínez Bravo ${ }^{64}$, M. Mastrodicasa ${ }^{57,46}$, H.J. Mathes ${ }^{41}$, J. Matthews ${ }^{88}$, G. Matthiae ${ }^{62,51}$, E. Mayotte ${ }^{38}$, P.O. Mazur ${ }^{a}$, G. MedinaTanco $^{68}$, D. Melo $^{8}$, A. Menshikov ${ }^{40}$, K.-D. Merenda ${ }^{86}$, S. Michal ${ }^{33}$, M.I. Micheletti ${ }^{6}$, L. Miramonti ${ }^{59,49}$, S. Mollerach ${ }^{1}$, F. Montanet ${ }^{36}$, C. Morello ${ }^{54,52}$, M. Mostafá ${ }^{91}$, A.L. Müller ${ }^{8}$, M.A. Muller ${ }^{22}$, K. Mulrey ${ }^{15}$, R. Mussa ${ }^{52}$, M. Muzio ${ }^{90}$, W.M. Namasaka ${ }^{38}$, A. Nasr-Esfahani ${ }^{38}$, L. Nellen ${ }^{68}$, M. Niculescu-Oglinzanu ${ }^{73}$, M. Niechciol ${ }^{44}$, D. Nitz ${ }^{89}$, D. Nosek ${ }^{31}$, V. Novotny ${ }^{31}$, L. Nožka ${ }^{33}$, A Nucita ${ }^{56,48}$, L.A. Núñez ${ }^{30}$, M. Palatka ${ }^{32}$, J. Pallotta ${ }^{2}$, P. Papenbreer ${ }^{38}$, G. Parente ${ }^{79}$, A. Parra ${ }^{64}$, J. Pawlowsky ${ }^{38}$, M. Pech ${ }^{32}$, F. Pedreira ${ }^{79}$, J. Pȩkala ${ }^{70}$, R. Pelayo ${ }^{65}$, J. Peña-Rodriguez ${ }^{30}$, E.E. Pereira Martins ${ }^{39,8}$, J. Perez Armand ${ }^{21}$, C. Pérez Bertolli $^{8,41}$, M. Perlin ${ }^{8,41}$, L. Perrone ${ }^{56,48}$, S. Petrera ${ }^{45,46}$, T. Pierog ${ }^{41}$, M. Pimenta ${ }^{72}$, V. Pirronello ${ }^{58,47}$, M. Platino ${ }^{8}$, B. Pont $^{80}$, M. Pothast ${ }^{81,80}$, P. Privitera ${ }^{92}$, M. Prouza ${ }^{32}$, A. Puyleart ${ }^{89}$, S. Querchfeld ${ }^{38}$, J. Rautenberg ${ }^{38}$, D. Ravignani ${ }^{8}$, M. Reininghaus ${ }^{41,8}$, J. Ridky ${ }^{32}$, F. Riehn ${ }^{72}$, M. Risse ${ }^{44}$, V. Rizi ${ }^{57,46}$, W. Rodrigues de Carvalho ${ }^{21}$, J. Rodriguez Rojo ${ }^{11}$, M.J. Roncoroni ${ }^{8}$, S. Rossoni ${ }^{43}$, M. Roth ${ }^{41}$, E. Roulet ${ }^{1}$, A.C. Rovero ${ }^{5}$, P. Ruehl ${ }^{44}$, A. Saftoiu ${ }^{73}$, F. Salamida ${ }^{57,46}$, H. Salazar ${ }^{64}$, G. Salina ${ }^{51}$, J.D. Sanabria Gomez ${ }^{30}$, F. Sánchez ${ }^{8}$, E.M. Santos ${ }^{21}$, E. Santos ${ }^{32}$, F. Sarazin ${ }^{86}$, R. Sarmento ${ }^{72}$, C. Sarmiento-Cano ${ }^{8}$, R. Sato ${ }^{11}$, 
P. Savina ${ }^{56,48,34,94}$, C.M. Schäfer ${ }^{41}$, V. Scherini ${ }^{56,48}$, H. Schieler ${ }^{41}$, M. Schimassek ${ }^{39,8}$, M. Schimp ${ }^{38}$, F. Schlüter ${ }^{41,8}$, D. Schmidt ${ }^{39}$, O. Scholten ${ }^{84,15}$, P. Schovánek ${ }^{32}$, F.G. Schröder ${ }^{93,41}$, S. Schröder ${ }^{38}$, J. Schulte ${ }^{42}$, S.J. Sciutto ${ }^{4}$, M. Scornavacche ${ }^{8,41}$, A. Segreto ${ }^{53,47}$, S. Sehgal ${ }^{38}$, R.C. Shellard ${ }^{16}$, G. Sigl ${ }^{43}$, G. Silli ${ }^{8,41}$, O. Sima ${ }^{73, f}$, R. Šmída ${ }^{92}$, P. Sommers ${ }^{91}$, J.F. Soriano ${ }^{87}$, J. Souchard ${ }^{36}$, R. Squartini ${ }^{10}$, M. Stadelmaier ${ }^{41,8}$, D. Stanca ${ }^{73}$, S. Staničc ${ }^{76}$, J. Stasielak ${ }^{70}$, P. Stassi ${ }^{36}$, A. Streich ${ }^{39,8}$, M. Suárez-Durán ${ }^{14}$, T. Sudholz ${ }^{13}$, T. Suomijärvi ${ }^{37}$, A.D. Supanitsky ${ }^{8}$, Z. Szadkowski ${ }^{71}$, A. Tapia ${ }^{29}$, C. Taricco ${ }^{63,52}$, C. Timmermans ${ }^{81,80}$, O. Tkachenko ${ }^{41}$, P. Tobiska ${ }^{32}$, C.J. Todero Peixoto ${ }^{19}$, B. Tomé ${ }^{72}$, Z. Torrès ${ }^{36}$, A. Travaini ${ }^{10}$, P. Travnicek ${ }^{32}$, C. Trimarelli ${ }^{57,46}$, M. Tueros ${ }^{4}$, R. Ulrich ${ }^{41}$, M. Unger ${ }^{41}$, L. Vaclavek ${ }^{33}$, M. Vacula ${ }^{33}$, J.F. Valdés Galicia ${ }^{68}$, L. Valore ${ }^{60,50}$, E. Varela ${ }^{64}$, A. Vásquez-Ramírez ${ }^{30}$, D. Veberič ${ }^{41}$, C. Ventura ${ }^{27}$, I.D. Vergara Quispe ${ }^{4}$, V. Verzi ${ }^{51}$, J. Vicha ${ }^{32}$, J. Vink ${ }^{83}$, S. Vorobiov ${ }^{76}$, H. Wahlberg ${ }^{4}$, C. Watanabe ${ }^{26}$, A.A. Watson ${ }^{c}$, M. Weber ${ }^{40}$, A. Weindl ${ }^{41}$, L. Wiencke ${ }^{86}$, H. Wilczyński ${ }^{70}$, M. Wirtz ${ }^{42}$, D. Wittkowski ${ }^{38}$, B. Wundheiler ${ }^{8}$, A. Yushkov $^{32}$, O. Zapparrata ${ }^{14}$, E. Zas ${ }^{79}$, D. Zavrtanik ${ }^{76,77}$, M. Zavrtanik ${ }^{77,76}$, L. Zehrer ${ }^{76}$

${ }^{1}$ Centro Atómico Bariloche and Instituto Balseiro (CNEA-UNCuyo-CONICET), San Carlos de Bariloche, Argentina

${ }^{2}$ Centro de Investigaciones en Láseres y Aplicaciones, CITEDEF and CONICET, Villa Martelli, Argentina

${ }^{3}$ Departamento de Física and Departamento de Ciencias de la Atmósfera y los Océanos, FCEyN, Universidad de Buenos Aires and CONICET, Buenos Aires, Argentina

${ }^{4}$ IFLP, Universidad Nacional de La Plata and CONICET, La Plata, Argentina

${ }^{5}$ Instituto de Astronomía y Física del Espacio (IAFE, CONICET-UBA), Buenos Aires, Argentina

${ }^{6}$ Instituto de Física de Rosario (IFIR) - CONICET/U.N.R. and Facultad de Ciencias Bioquímicas y Farmacéuticas U.N.R., Rosario, Argentina

${ }^{7}$ Instituto de Tecnologías en Detección y Astropartículas (CNEA, CONICET, UNSAM), and Universidad Tecnológica Nacional - Facultad Regional Mendoza (CONICET/CNEA), Mendoza, Argentina

${ }^{8}$ Instituto de Tecnologías en Detección y Astropartículas (CNEA, CONICET, UNSAM), Buenos Aires, Argentina

${ }^{9}$ International Center of Advanced Studies and Instituto de Ciencias Físicas, ECyT-UNSAM and CONICET, Campus Miguelete - San Martín, Buenos Aires, Argentina

${ }^{10}$ Observatorio Pierre Auger, Malargüe, Argentina

${ }^{11}$ Observatorio Pierre Auger and Comisión Nacional de Energía Atómica, Malargüe, Argentina

12 Universidad Tecnológica Nacional - Facultad Regional Buenos Aires, Buenos Aires, Argentina

${ }^{13}$ University of Adelaide, Adelaide, S.A., Australia

14 Université Libre de Bruxelles (ULB), Brussels, Belgium

15 Vrije Universiteit Brussels, Brussels, Belgium

${ }^{16}$ Centro Brasileiro de Pesquisas Fisicas, Rio de Janeiro, RJ, Brazil

${ }^{17}$ Centro Federal de Educação Tecnológica Celso Suckow da Fonseca, Nova Friburgo, Brazil

${ }^{18}$ Instituto Federal de Educação, Ciência e Tecnologia do Rio de Janeiro (IFRJ), Brazil

${ }^{19}$ Universidade de São Paulo, Escola de Engenharia de Lorena, Lorena, SP, Brazil

${ }^{20}$ Universidade de São Paulo, Instituto de Física de São Carlos, São Carlos, SP, Brazil

${ }^{21}$ Universidade de São Paulo, Instituto de Física, São Paulo, SP, Brazil

${ }^{22}$ Universidade Estadual de Campinas, IFGW, Campinas, SP, Brazil

${ }^{23}$ Universidade Estadual de Feira de Santana, Feira de Santana, Brazil

${ }^{24}$ Universidade Federal do ABC, Santo André, SP, Brazil

${ }^{25}$ Universidade Federal do Paraná, Setor Palotina, Palotina, Brazil

${ }^{26}$ Universidade Federal do Rio de Janeiro, Instituto de Física, Rio de Janeiro, RJ, Brazil

${ }^{27}$ Universidade Federal do Rio de Janeiro (UFRJ), Observatório do Valongo, Rio de Janeiro, RJ, Brazil

${ }^{28}$ Universidade Federal Fluminense, EEIMVR, Volta Redonda, RJ, Brazil

${ }^{29}$ Universidad de Medellín, Medellín, Colombia

${ }^{30}$ Universidad Industrial de Santander, Bucaramanga, Colombia

${ }^{31}$ Charles University, Faculty of Mathematics and Physics, Institute of Particle and Nuclear Physics, Prague, Czech Republic

32 Institute of Physics of the Czech Academy of Sciences, Prague, Czech Republic 
${ }^{33}$ Palacky University, RCPTM, Olomouc, Czech Republic

34 CNRS/IN2P3, IJCLab, Université Paris-Saclay, Orsay, France

${ }^{35}$ Laboratoire de Physique Nucléaire et de Hautes Energies (LPNHE), Sorbonne Université, Université de Paris, CNRSIN2P3, Paris, France

${ }^{36}$ Univ. Grenoble Alpes, CNRS, Grenoble Institute of Engineering Univ. Grenoble Alpes, LPSC-IN2P3, 38000 Grenoble, France

${ }^{37}$ Université Paris-Saclay, CNRS/IN2P3, IJCLab, Orsay, France

38 Bergische Universität Wuppertal, Department of Physics, Wuppertal, Germany

${ }^{39}$ Karlsruhe Institute of Technology (KIT), Institute for Experimental Particle Physics, Karlsruhe, Germany

${ }^{40}$ Karlsruhe Institute of Technology (KIT), Institut für Prozessdatenverarbeitung und Elektronik, Karlsruhe, Germany

${ }^{41}$ Karlsruhe Institute of Technology (KIT), Institute for Astroparticle Physics, Karlsruhe, Germany

${ }^{42}$ RWTH Aachen University, III. Physikalisches Institut A, Aachen, Germany

43 Universität Hamburg, II. Institut für Theoretische Physik, Hamburg, Germany

${ }^{44}$ Universität Siegen, Department Physik - Experimentelle Teilchenphysik, Siegen, Germany

${ }^{45}$ Gran Sasso Science Institute, L'Aquila, Italy

46 INFN Laboratori Nazionali del Gran Sasso, Assergi (L'Aquila), Italy

${ }^{47}$ INFN, Sezione di Catania, Catania, Italy

${ }^{48}$ INFN, Sezione di Lecce, Lecce, Italy

${ }^{49}$ INFN, Sezione di Milano, Milano, Italy

${ }^{50}$ INFN, Sezione di Napoli, Napoli, Italy

${ }^{51}$ INFN, Sezione di Roma "Tor Vergata", Roma, Italy

52 INFN, Sezione di Torino, Torino, Italy

53 Istituto di Astrofisica Spaziale e Fisica Cosmica di Palermo (INAF), Palermo, Italy

54 Osservatorio Astrofisico di Torino (INAF), Torino, Italy

55 Politecnico di Milano, Dipartimento di Scienze e Tecnologie Aerospaziali, Milano, Italy

56 Università del Salento, Dipartimento di Matematica e Fisica "E. De Giorgi”, Lecce, Italy

57 Università dell'Aquila, Dipartimento di Scienze Fisiche e Chimiche, L'Aquila, Italy

58 Università di Catania, Dipartimento di Fisica e Astronomia, Catania, Italy

${ }^{59}$ Università di Milano, Dipartimento di Fisica, Milano, Italy

${ }^{60}$ Università di Napoli "Federico II", Dipartimento di Fisica "Ettore Pancini”, Napoli, Italy

${ }^{61}$ Università di Palermo, Dipartimento di Fisica e Chimica "E. Segrè", Palermo, Italy

62 Università di Roma "Tor Vergata", Dipartimento di Fisica, Roma, Italy

${ }^{63}$ Università Torino, Dipartimento di Fisica, Torino, Italy

${ }^{64}$ Benemérita Universidad Autónoma de Puebla, Puebla, México

${ }^{65}$ Unidad Profesional Interdisciplinaria en Ingeniería y Tecnologías Avanzadas del Instituto Politécnico Nacional (UPIITA-IPN), México, D.F., México

66 Universidad Autónoma de Chiapas, Tuxtla Gutiérrez, Chiapas, México

${ }^{67}$ Universidad Michoacana de San Nicolás de Hidalgo, Morelia, Michoacán, México

${ }^{68}$ Universidad Nacional Autónoma de México, México, D.F., México

${ }^{69}$ Universidad Nacional de San Agustin de Arequipa, Facultad de Ciencias Naturales y Formales, Arequipa, Peru

${ }^{70}$ Institute of Nuclear Physics PAN, Krakow, Poland

${ }^{71}$ University of Łódź, Faculty of High-Energy Astrophysics, Łódź, Poland

${ }^{72}$ Laboratório de Instrumentação e Física Experimental de Partículas - LIP and Instituto Superior Técnico - IST, Universidade de Lisboa - UL, Lisboa, Portugal

73 "Horia Hulubei” National Institute for Physics and Nuclear Engineering, Bucharest-Magurele, Romania

${ }^{74}$ Institute of Space Science, Bucharest-Magurele, Romania

75 University Politehnica of Bucharest, Bucharest, Romania

76 Center for Astrophysics and Cosmology (CAC), University of Nova Gorica, Nova Gorica, Slovenia

${ }^{77}$ Experimental Particle Physics Department, J. Stefan Institute, Ljubljana, Slovenia

78 Universidad de Granada and C.A.F.P.E., Granada, Spain

${ }^{79}$ Instituto Galego de Física de Altas Enerxías (IGFAE), Universidade de Santiago de Compostela, Santiago de Compostela, Spain 
${ }^{80}$ IMAPP, Radboud University Nijmegen, Nijmegen, The Netherlands

${ }^{81}$ Nationaal Instituut voor Kernfysica en Hoge Energie Fysica (NIKHEF), Science Park, Amsterdam, The Netherlands

82 Stichting Astronomisch Onderzoek in Nederland (ASTRON), Dwingeloo, The Netherlands

${ }^{83}$ Universiteit van Amsterdam, Faculty of Science, Amsterdam, The Netherlands

${ }^{84}$ University of Groningen, Kapteyn Astronomical Institute, Groningen, The Netherlands

85 Case Western Reserve University, Cleveland, OH, USA

86 Colorado School of Mines, Golden, CO, USA

${ }^{87}$ Department of Physics and Astronomy, Lehman College, City University of New York, Bronx, NY, USA

${ }^{88}$ Louisiana State University, Baton Rouge, LA, USA

${ }^{89}$ Michigan Technological University, Houghton, MI, USA

${ }^{90}$ New York University, New York, NY, USA

${ }^{91}$ Pennsylvania State University, University Park, PA, USA

92 University of Chicago, Enrico Fermi Institute, Chicago, IL, USA

93 University of Delaware, Department of Physics and Astronomy, Bartol Research Institute, Newark, DE, USA

94 University of Wisconsin-Madison, Department of Physics and WIPAC, Madison, WI, USA

${ }^{a}$ Fermi National Accelerator Laboratory, Fermilab, Batavia, IL, USA

${ }^{b}$ Max-Planck-Institut für Radioastronomie, Bonn, Germany

${ }^{c}$ School of Physics and Astronomy, University of Leeds, Leeds, United Kingdom

${ }^{d}$ Colorado State University, Fort Collins, CO, USA

$e^{e}$ now at Hakubi Center for Advanced Research and Graduate School of Science, Kyoto University, Kyoto, Japan

$f$ also at University of Bucharest, Physics Department, Bucharest, Romania 